\title{
Function and culture requirements of snow leopard (Panthera uncia) spermatozoa in vitro
}

\author{
T. L. Roth, J. G. Howard, A. M. Donoghue, W. F. Swanson and \\ D. E. Wildt
}

National Zoological Park, Smithsonian Institution, Washington, DC 20008, USA

\begin{abstract}
Electroejaculates from eight snow leopards were used to determine how the motility of spermatozoa was influenced by (i) type of media (Ham's F10, PBS, human tubal fluid or RPMI-1640); (ii) holding temperature $\left(23^{\circ} \mathrm{C}\right.$ versus $\left.37^{\circ} \mathrm{C}\right)$; (iii) washing of spermatozoa and (iv) a sperm metabolic enhancer, pentoxifylline. The duration of sperm motility was assessed by evaluating samples in each treatment every hour for $6 \mathrm{~h}$ and a sperm motility index (a value combining percentage sperm motility and rate of forward progression) calculated. Spermatozoa from the Ham's F10, PBS and PBS plus pentoxifylline treatments were also co-incubated with zona-intact, domestic cat eggs that were fixed and evaluated for spermatozoa bound to the zona pellucida, penetrating the outer and inner layers of the zona pellucida and within the perivitelline space. During the $6 \mathrm{~h}$ co-incubation, the sperm motility index in PBS with pentoxifylline was greater $(P<0.05)$ than in PBS alone which, in turn, was greater $(P<0.05)$ than in the other three test media. Washing the spermatozoa enhanced $(P<0.05)$ motility in both PBS and PBS plus pentoxifylline relative to unwashed samples, but there was no effect $(P>0.05)$ of holding temperature. Pentoxifylline supplementation enhanced $(P<0.05)$ the proportion of cat eggs with bound, but not penetrated, snow leopard spermatozoa. Only one to three of the 120 total cat eggs/treatment group had snow leopard spermatozoa in the inner layer of the zona pellucida, and there were no spermatozoa in the perivitelline space. Failure of sperm penetration did not appear related to an inadequacy in the culture system because nine of 27 snow leopard eggs co-incubated in PBS with conspecific spermatozoa cleaved in vitro and nine of the 18 unfertilized eggs contained spermatozoa in the perivitelline space. The snow leopard appears unique among felid species in that (i) sperm viability is highly sensitive to type of culture medium, (ii) sperm motility and function in vitro appear enhanced by a simple rather than complex culture medium and (iii) there is a mechanism that prevents these spermatozoa from fully penetrating heterologous, salt-stored, domestic cat eggs.
\end{abstract}

\section{Introduction}

The snow leopard (Panthera uncia), one of the great cats, is formally listed by the Convention on International Trade in Endangered Species as endangered by extinction (CITES, 1984). Survival of this and the other 35 nondomestic felid species threatened by extirpation depends upon an array of conservation strategies including intensive research and captive breeding (Wildt et al., 1992a, b). One component of a captive breeding programme involves assisted reproductive techniques to enhance propagation and maintain genetic diversity. However, the potential of artificial insemination, in vitro fertilization (IVF) or gamete cryopreservation depends upon understanding the fundamental biology associated with optimal gamete processing.

Sperm viability in the domestic cat (Felis catus), cheetah (Acinonyx jubatus) and tiger (P. tigris) is sustained in vitro in

Received 19 October 1993.
Ham's F10, a complex tissue culture medium that also supports successful IVF of conspecific eggs matured in vivo in these species (Donoghue et al., 1990, 1992a; Johnston et al., 1991a; Howard et al., 1993). Similarly, a heterologous egg penetration assay in Ham's F10 in vitro has proved useful for assessing sperm functionality in the cheetah (Donoghue et al., 1992a) and tiger (Donoghue et al., 1992c) because these spermatozoa bind and penetrate the zona pellucida of salt-stored, domestic cat eggs. In a preliminary study, we found that snow leopard spermatozoa from high quality ejaculates inexplicably exhibited short-lived motility in Ham's F10 $(<3 \mathrm{~h})$.

Developing efficient assisted reproduction requires a medium supportive of snow leopard sperm viability and function in vitro. Information gained from examining functionality and culture requirements of $P$. uncia spermatozoa may also prove useful for understanding and optimizing gamete interaction in other species within the Felidae. Our general aim was to identify a culture medium that would support the function of snow leopard spermatozoa in vitro while examining various 
processing factors potentially responsible for the unusually short lifespan of spermatozoa observed in the preliminary study. Our three specific objectives were to (i) compare the duration of snow leopard sperm motility in complex versus simple culture media, at $23^{\circ} \mathrm{C}$ versus $37^{\circ} \mathrm{C}$ and in unwashed versus washed samples, (ii) assess the ability of these spermatozoa to interact with domestic cat eggs in a heterologous sperm functionality assay and (iii) evaluate the influence of the sperm metabolic stimulator, pentoxifylline, on sperm motility, longevity in vitro and ability to penetrate the domestic cat zona pellucida.

\section{Materials and Methods}

\section{Animals}

Eight adult snow leopards (3-13 years of age; $36-51 \mathrm{~kg}$ each) served as semen donors. All were born in captivity and maintained at the Greater Baton Rouge Zoo (Baton Rouge, LA), Jackson Zoological Park (Jackson, MS), The Wildlife Conservation Society (New York), Seneca Park Zoo (Rochester, NY) or the Woodland Park Zoological Gardens (Seattle, WA). The study was conducted during April and May.

\section{Semen collection and processing}

A surgical plane of anaesthesia for semen collection was induced with tiletamine and zolazepam $\left(5 \mathrm{mg} \mathrm{kg}^{-1}\right.$ body weight i.m.; Telazol ${ }^{\mathbb{R}}$, A. H. Robins Co., Richmond, VA). Semen was collected by standardized electroejaculation (Wildt et al., 1983; Howard et al., 1986a) using a rectal probe $(2.3 \mathrm{~cm}$ diameter) with three longitudinal electrodes. In brief, each animal received three series of electrical stimuli ( 80 stimuli total; range $2-5$ volts) over a period of about $30 \mathrm{~min}$. After each stimulus series, total fluid volume was recorded and $5 \mu \mathrm{l}$ evaluated under phase-contrast microscopy $(\times 25)$ for subjective estimates of percentage sperm motility $(0-100 \%)$ and progressive motility on a $0-5$ scale ( 0 : no movement; 5 : rapid forward progression; Howard et al., 1986a). The $\mathrm{pH}$ was estimated by evaluating the colour change after pipetting $5 \mu \mathrm{l}$ of semen onto an indicator strip (EM Science, Gibbstown, NJ). Semen collected during Series 1 and 3 was diluted immediately with an equal volume of Dulbecco's phosphate-buffered saline (PBS; Irvine Scientific, Santa Ana, CA) containing 10\% fetal calf serum (FCS; Irvine Scientific), $0.011 \mathrm{mg}$ pyruvate $\mathrm{ml}^{-1}$ (Sigma Chemical Company, St Louis, MO), $100 \mathrm{U}$ penicillin $\mathrm{ml}^{-1}$ (Sigma) and $100 \mu \mathrm{g}$ streptomycin $\mathrm{ml}^{-1}$ (Sigma). After measuring total volume for Series 2,50 $\mu \mathrm{l}$ aliquots of undiluted semen were transferred into multiple sterile microcentrifuge tubes (Lab Source Inc., Chicago, IL). An additional $10 \mu \mathrm{l}$ aliquot was placed in $100 \mu \mathrm{l}$ of $0.3 \%$ glutaraldehyde fixative for detailed morphological analysis (100 spermatozoa per aliquot, $\times 1000$ under oil) according to previous criteria (Wildt et al., 1983; Howard et al., 1986a). The remaining Series 2 semen was diluted with an equal volume of PBS and then combined with Series 1 and 3 samples. The total number of spermatozoa ejaculated was determined using a haemocytometer with $10 \mu \mathrm{l}$ of this combined, diluted semen (Howard et al., 1986a).
Semen treatments and evaluations of sperm motility over time

Each microcentrifuge tube containing $50 \mu \mathrm{l}$ of semen was assigned to one of three studies conducted simultaneously, and was diluted $1: 3(\mathrm{v}: \mathrm{v})$ with the appropriate medium required per study. Study $I$ was designed to compare the effects of simple versus complex media and the treatments were: (i) Ham's F10 medium (Irvine Scientific); (ii) PBS; (iii) Roswell Park Memorial Institute-1640 medium (RPMI: Sigma) and (iv) human tubal fluid (Irvine Scientific; HTF). The medium in each of these treatment groups was maintained at $37^{\circ} \mathrm{C}$.

In Study 2, the effect of incubation temperature and a sperm metabolic enhancer were examined by comparing the following treatments: (i) $\mathrm{PBS}$ at $37^{\circ} \mathrm{C}$ (PBS-37); (ii) $\mathrm{PBS}$ at $23^{\circ} \mathrm{C}$ (PBS-23); (iii) PBS supplemented with $1 \mathrm{mg}$ pentoxifylline $\mathrm{ml}^{-1}$ (Sigma) at $23^{\circ} \mathrm{C}$ (PBSP-23); and (iv) PBS supplemented with $1 \mathrm{mg}$ pentoxifylline $\mathrm{ml}^{-1}$ at $37^{\circ} \mathrm{C}$ (PBSP-37).

In Study 3, the effects of washing spermatozoa and the sperm metabolic enhancer were examined by comparing: (i) PBS; (ii) PBS centrifuged ( $150 \mathrm{~g}, 10 \mathrm{~min})$, supernatant discarded and sperm pellet resuspended in $100 \mu \mathrm{PBS}$ (PBS-WS); (iii) PBSP; and (iv) PBSP centrifuged ( $150 \mathrm{~g}, 10 \mathrm{~min})$, supernatant discarded and sperm pellet resuspended in $100 \mu \mathrm{l}$ PBSP (PBSPWS). The medium in each of these treatment groups was maintained at $23^{\circ} \mathrm{C}$.

All media were supplemented with 10\% FCS, $0.011 \mathrm{mg}$ pyruvate $\mathrm{ml}^{-1}, 100 \mathrm{U}$ penicillin $\mathrm{ml}^{-1}$ and $100 \mu \mathrm{g}$ streptomycin $\mathrm{ml}^{-1}$. Immediately after dilution, samples from each treatment were evaluated for percentage sperm motility and forward progressive motility (time $0 \mathrm{~h}$ ). Sperm concentration was determined and each treatment diluted to $5 \times 10^{\circ}$ motile spermatozoa $\mathrm{ml}^{-1}$. Treatments maintained at $37^{\circ} \mathrm{C}$ were incubated on a slide warmer and protected from light under a plastic chamber in a $5 \% \mathrm{CO}_{2}$ and air atmosphere. Room temperature treatments $\left(23^{\circ} \mathrm{C}\right)$ were maintained in a covered Styrofoam box in a $100 \%$ air atmosphere. All treatment groups were re-evaluated for percentage sperm motility and forward progressive motility at $0.5 \mathrm{~h}$ and then every hour for $6 \mathrm{~h}$ (0.5 h-6.0 h).

\section{Salt-stored egg penetration assay}

Immature eggs were recovered from domestic cat ovaries following ovariohysterectomy (Johnston et al., 1989; Andrews et al., 1992). Briefly, ovaries maintained in PBS at $5^{\circ} \mathrm{C}$ for up to $48 \mathrm{~h}$ after ovariohysterectomy were placed in Eagle's minimum essential medium (MEM; Sigma) supplemented with 5\% FCS and punctured repeatedly with a 22 gauge needle to release cumulus-egg complexes. Darkly pigmented eggs were rinsed three times and then cultured in MEM containing $5 \%$ FCS, $0.284 \mathrm{mg}$ L-glutamine $\mathrm{ml}^{-1}, 0.011 \mathrm{mg}$ pyruvate $\mathrm{ml}^{-1}$, $10.0 \mu \mathrm{g} \mathrm{LH} \mathrm{ml}^{-1}$ (NIADDK-oLH-25 AFP-5551B), 1.0 $\mu \mathrm{g} \mathrm{FSH}$ $\mathrm{ml}^{-1}$ (NIADDK-oFSH-17 AFP-6446C), $1.0 \mu \mathrm{g}$ oestradiol

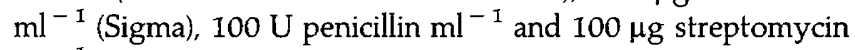
$\mathrm{ml}^{-1}$. After $48-52 \mathrm{~h}$ in culture, eggs were treated with $0.2 \%$ hyaluronidase (Sigma) in MEM for $15 \mathrm{~min}\left(38^{\circ} \mathrm{C}\right)$ and mechanically pipetted to remove any cumulus cells adhered to the zona pellucida. Eggs were rinsed through three drops of maturation medium and transferred into vials containing a hypertonic salt-storage solution (Andrews et al., 1992) consisting of 
$0.5 \mathrm{~mol}\left(\mathrm{NH}_{4}\right)_{2} \mathrm{SO}_{4} \mathrm{l}^{-1}, 0.75 \mathrm{~mol} \mathrm{MgCl}_{2} \mathrm{l}^{-1}, 0.2 \mathrm{mmol} \mathrm{ZnCl}_{2}$ $\mathrm{l}^{-1}, 0.1 \mathrm{mg}$ polyvinyl alcohol $\mathrm{ml}^{-1}$ (Sigma) and $40 \mathrm{mmol}$ Hepes buffer $1^{-1}$ (Sigma; $\mathrm{pH} 7.4$ ) and then stored at $4^{\circ} \mathrm{C}$.

The effects of simple versus complex media and the chemical sperm enhancer pentoxifylline on sperm function were compared by conducting the egg penetration assay in the Ham's F10, PBS-37 and PBSP-37 treatment groups. For each ejaculate, 45 salt-stored eggs were recovered from the salt storage solution and prepared for insemination. Fifteen eggs were equilibrated in medium from each treatment group (Ham's F10, PBS-37 and PBSP-37) by incubating the eggs twice ( $1 \mathrm{~h}$ per incubation) in $100 \mu$ l drops of the respective medium covered with oil. Eggs were then transferred into $90 \mu \mathrm{l}$ drops of their respective media and inseminated with $10 \mu \mathrm{l}$ semen $\left(5 \times 10^{4}\right.$ motile spermatozoa) from Ham's F10, PBS-37 and PBSP-37 treatments. After co-incubation for $6 \mathrm{~h}\left(5 \% \mathrm{CO}_{2} ; 37^{\circ} \mathrm{C}\right)$ with spermatozoa, eggs were fixed in $10 \%$ buffered formalin and stored at $5^{\circ} \mathrm{C}$ until evaluated.

Penetration of eggs (about 120 per treatment group) was evaluated using differential interference contrast microscopy ( $\times 320$; Andrews ef al., 1992; Howard et al., 1993). Each egg was assessed for the number of (i) spermatozoa bound to the outer zona; (ii) sperm heads within the outer half of the zona; (iii) sperm heads within the inner half of the zona; and (iv) spermatozoa within the perivitelline space.

\section{Conspecific egg collection and IVF}

For comparative purposes, IVF was also performed using eggs recovered from three female snow leopards given 800 iu pregnant mares' serum gonadotrophin i.m. (Sigma) followed $84 \mathrm{~h}$ later (Donoghue et al., 1992b) with 400 iu hCG i.m. (Sigma). Twenty-five hours after administering hCG, females were subjected to laparoscopy and eggs aspirated from preovulatory ovarian follicles ( $\geq 2 \mathrm{~mm}$ in diameter) using a 22 gauge needle (Goodrowe et al., 1988). Follicular contents were collected into a tube containing Ham's F10 supplemented with $40 \mathrm{iu}$ heparin $\mathrm{ml}^{-1}\left(37^{\circ} \mathrm{C}\right)$. Eggs were transferred into a Petri dish containing $3 \mathrm{ml} \mathrm{Ham}$ 's F10, washed twice, and good-toexcellent quality, mature eggs (darkly pigmented, uniform eggs with an expanded cumulus; Goodrowe et al., 1988; Johnston et al., 1991a) were placed into a $100 \mu \mathrm{l}$ drop of Ham's F10 overlaid with oil and maintained in $5 \% \mathrm{CO}_{2}$ and air until insemination. Immediately before exposure to spermatozoa, eggs were rinsed through two $100 \mu \mathrm{l}$ drops of PBS and then transferred to a $90 \mu \mathrm{l}$ drop of PBS. Spermatozoa were processed and eggs inseminated (as described for salt-stored cat eggs). Eight hours later, eggs were rinsed free of cumulus cells and transferred into Ham's F10 culture drops overlaid with oil and maintained at $38{ }^{\circ} \mathrm{C}$ in humidified $5 \% \mathrm{CO}_{2}$ and air. Eggs were examined at 30 and $54 \mathrm{~h}$ for cleavage, and all noncleaving eggs were fixed in $10 \%$ buffered formalin and assessed for sperm penetration of the zona as described for the salt-stored cat eggs.

\section{Statistical analysis}

Sperm motility index longevity data were analysed in a $4 \times 8$ (media $\times$ time) factorial arrangement analysis of variance
Table 1. Characteristics of snow leopard ejaculates

\begin{tabular}{lr}
\hline Parameters & $\begin{array}{r}\text { Mean } \pm \text { SEM } \\
(n=8 \text { males })\end{array}$ \\
\hline Ejaculate volume $(\mathrm{ml})$ & $2.6 \pm 0.3$ \\
$\mathrm{pH}$ & $8.6 \pm 0.1$ \\
Total spermatozoa per ejaculate $\left(\times 10^{6}\right)$ & $277.3 \pm 71.8$ \\
Percentage sperm motility & $78.1 \pm 2.1$ \\
Sperm forward progressive motility $(0-5)^{\mathrm{a}}$ & $4.3 \pm 0.1$ \\
Sperm motility index & $81.6 \pm 1.9$ \\
Morphologically normal spermatozoa $(\%)$ & $63.3 \pm 3.0$ \\
Morphologically abnormal spermatozoa $(\%)$ & \\
$\quad$ Abnormal head & $1.2 \pm 0.3$ \\
Abnormal acrosome & $5.7 \pm 2.5$ \\
Abnormal midpiece & $1.2 \pm 0.3$ \\
Coiled flagellum & $2.0 \pm 0.5$ \\
Bent midpiece & $4.2 \pm 1.0$ \\
Bent flagellum & $11.6 \pm 1.6$ \\
Cytoplasmic droplet & $10.1 \pm 2.2$ \\
Spermatid & $0.7 \pm 0.2$ \\
\hline
\end{tabular}

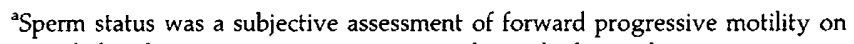
a graded scale: 0 , no movement to 5 , rapid, steady forward progression.

${ }^{b}$ Sperm motility index $=[\%$ motility $+($ progressive motility $\times 20)] \div 2$.

(ANOVA) using the general linear model procedures of the Statistical Analysis Systems (SAS) computer package (SAS User's Guide, 1985). Sperm motility index profiles over time were compared using polynomial regression analyses. Egg penetration data were analysed by ANOVA, and all treatment means were compared using Duncan's multiple range test. Chi square $\left(\chi^{2}\right)$ analysis was used to determine differences in the proportion of penetrated eggs among the treatment groups.

\section{Results}

Ejaculates containing spermatozoa were obtained from all eight snow leopards, and fresh ejaculate quality was high (Table 1). The total number of spermatozoa per ejaculate was $277 \times 10^{6}$ cells, more than $75 \%$ of spermatozoa were motile, fewer than $40 \%$ of spermatozoa were structurally malformed and most defects were cells with a bent flagellum or cytoplasmic droplet.

It was apparent from the steep negative slopes of the sperm motility index profiles that in vitro conditions were deleterious to sperm motility longevity regardless of dilution medium used (Study 1, Fig. 1). However, the sperm motility index for spermatozoa exposed to the simplest medium, PBS, was superior $(P<0.05)$ to that of spermatozoa in Ham's F10, HTF or RPMI. In addition, the sperm motility index profile slope for PBS followed a gradual, linear descent in contrast to the slopes of the other media that best fit quadratic functions. Although the sperm motility index at $1 \mathrm{~h}$ was greater $(P<0.05)$ for Ham's FIO than for RPMI and HTF, there were no overall profile differences $(P>0.05)$ among these three media.

During the $6 \mathrm{~h}$ incubation, sperm motility index profiles (magnitude, duration and rate of decline in slope) were not statistically different $(P>0.05)$ among the temperature groups tested (Study 2, data not shown). In contrast, washing the 


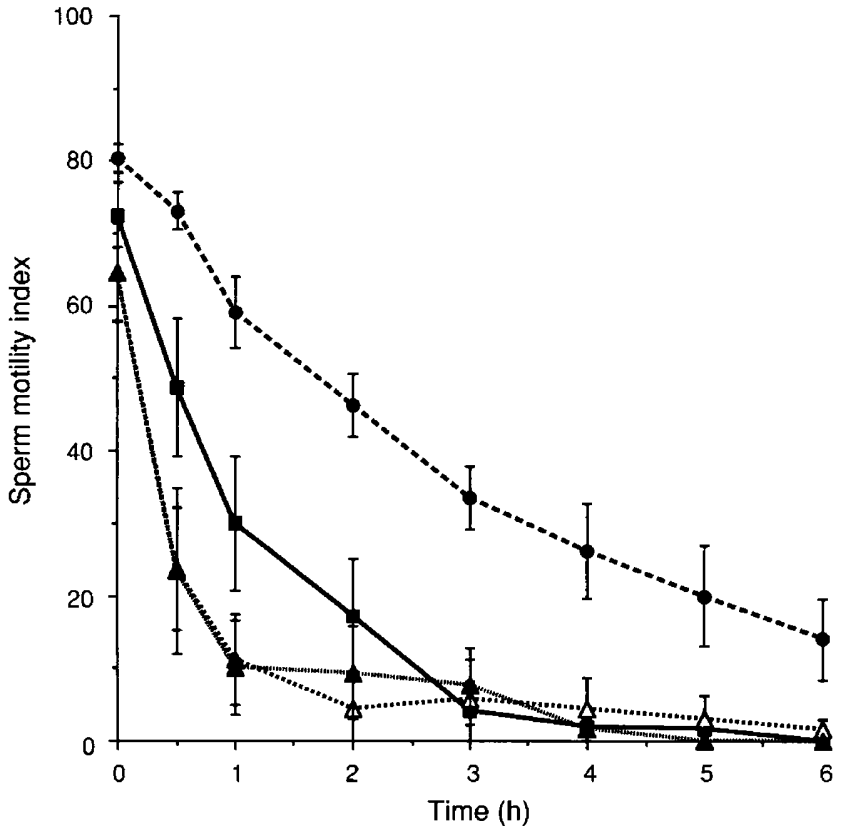

Fig. 1. Influence of four culture media on longevity of snow leopard spermatozoa in vitro at $37^{\circ} \mathrm{C}$. Values are means ( \pm SEM; $n=8$ ). PBS $(\bullet)$ enhanced $(P<0.05)$ the sperm motility index compared with

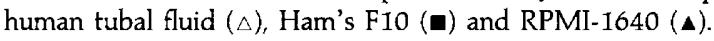

spermatozoa affected their motility (Fig. 2) with the overall amplitude of sperm motility index of washed spermatozoa enhanced $(P<0.05)$ compared with unwashed samples. However, the negative slopes of the PBS-WS and PBS curves were not different $(P>0.05)$, indicating a similar rate of decline in sperm motility during the $6 \mathrm{~h}$. Similar results were obtained when the sperm motility index over time for PBS was compared with that for PBSP. The metabolic stimulator pentoxifylline, when added to PBS, enhanced $(P<0.05)$ the magnitude of the sperm motility index profile compared with PBS alone (Fig. 2). However, sperm motility decreased at a similar rate $(P>0.05)$ in PBS $\left(-9.74 x: R^{2}=0.960\right)$ and PBSP $\left(-10.54 x: R^{2}=0.994\right)$, clearly demonstrating the inability of pentoxifylline to sustain a higher sperm motility index over time.

At least $70 \%$ of the salt-stored cat eggs were bound by snow leopard spermatozoa regardless of treatment (Study 4, Fig. 3a). Adding pentoxifylline to PBS enhanced $(P<0.05)$ the percentage of eggs bound with spermatozoa $(91.8 \pm 3.0) \mathrm{com}-$ pared with Ham's F10 (71.8 \pm 4.9$)$ and PBS (70.2 \pm 5.0$)$. The average number of bound spermatozoa per egg was also greater $(P<0.05)$ for PBSP than for Ham's F10 or PBS (Fig. 3b). The percentage of eggs containing spermatozoa in the outer zona pellucida ranged from 44.7 to $56.5 \%$ and was not different $(P>0.05)$ among treatments. The average number of spermatozoa penetrating the outer zona varied from 1.6 to 2.9 per egg and also was not different $(P>0.05)$ among treatments. For each treatment, almost all spermatozoa that penetrated stopped abruptly at the inner-outer zona interface (Fig. 4). Only one to three eggs contained spermatozoa in the inner zona, and no spermatozoa were observed in the perivitelline space of any cat egg in any treatment.

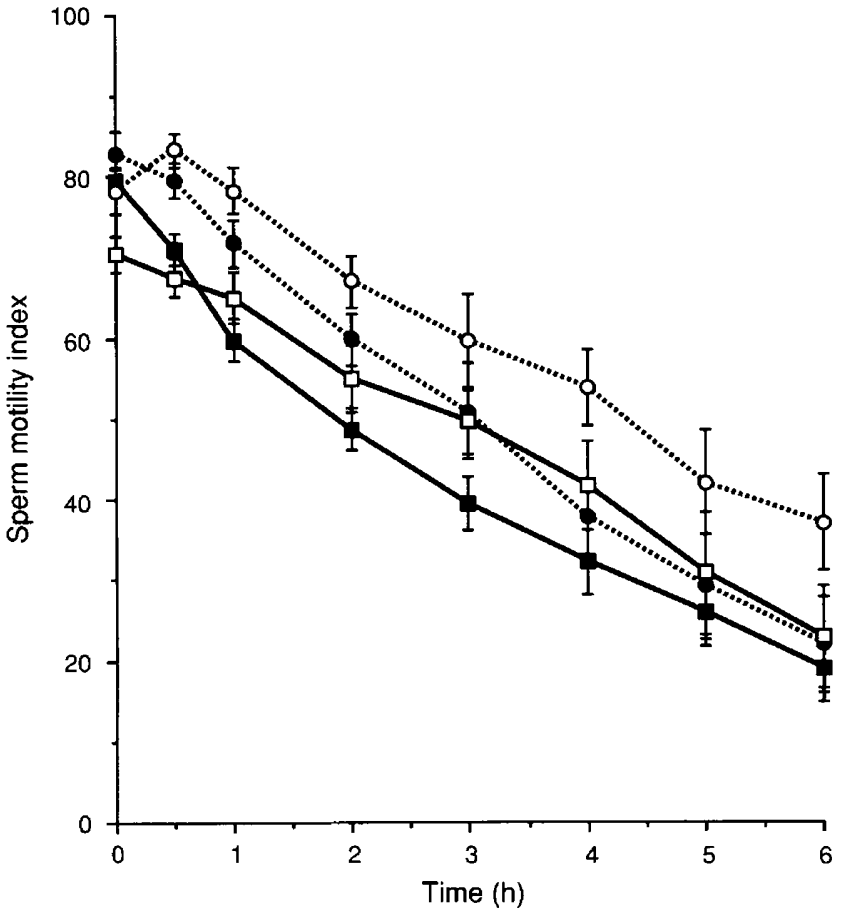

Fig. 2. Influence of washing the spermatozoa and a metabolic stimulator, pentoxifylline (PBSP), on lifespan of snow leopard spermatozoa maintained in culture in PBS. (匹) PBS, ( $\square$ ) PBS plus washing, (•) PBSP and (O) PBSP plus washing. Values are means ( \pm SEM; $n=8$ ). Both washing the spermatozoa and pentoxifylline supplementation enhanced $(P<0.05)$ overall sperm motility in PBS but did not affect $(P>0.05)$ the rate of its decline.

The conspecific co-culture of snow leopard spermatozoa and eggs was therefore important for determining whether the low incidence of inner zona penetration using cat eggs was related to suboptimal IVF conditions. Fifty-six eggs were recovered from the snow leopard donors. Twenty-seven of these met maturation criteria, were good-to-excellent in quality and were inseminated. Nine eggs cleaved to at least the two-cell stage, and four and three of these embryos developed to the five- to eight-cell and nine- to 16-cell stage, respectively, before being cryopreserved. Of the 18 eggs not cleaving, 17 had at least one spermatozoon in the outer zona, 15 contained spermatozoa in the inner zona, and nine had spermatozoa within the perivitelline space.

\section{Discussion}

The snow leopard was different from other felid species studied to date in that sperm longevity in vitro was supported by a simple (PBS) rather than a complex medium (Ham's F10). The ability to detect the apparent detrimental effects of Ham's F10 on snow leopard spermatozoa was due, in part, to the relatively high quality ejaculate produced by this species. Spermatozoa from other felids, including the cheetah and clouded leopard, exhibit reduced longevity in vitro, which is largely attributable to the poor ejaculate quality that is characteristic of these species (Wildt et al., 1983, 1986). In the felid taxon, the 
(a)

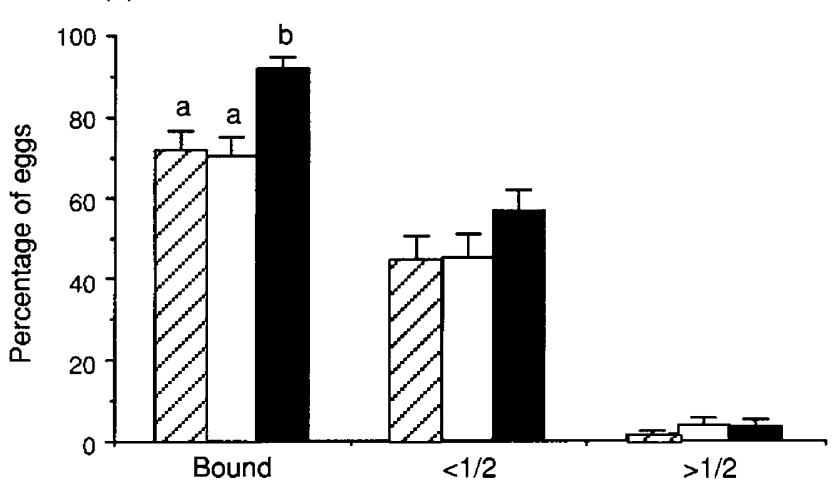

(b)

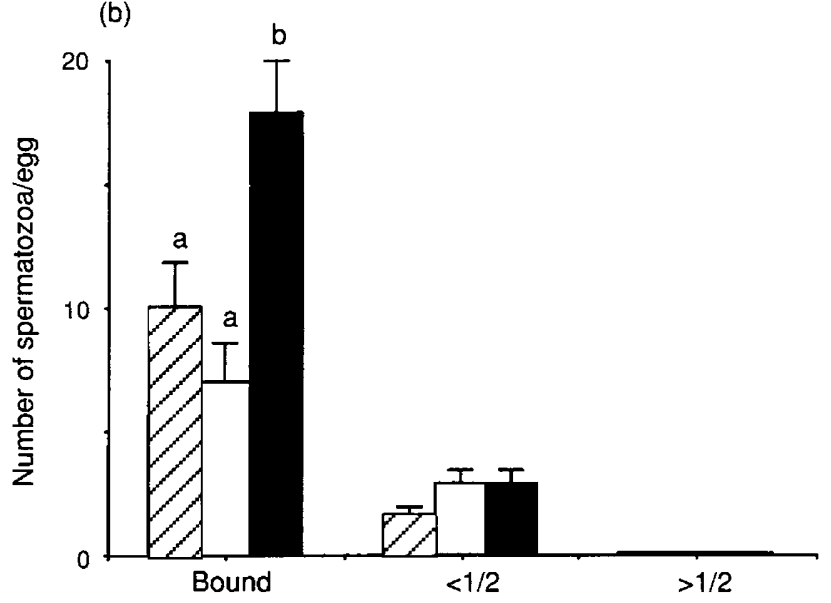

Fig. 3. Penetration of salt-stored domestric cat eggs by snow leopard spermatozoa from eight males. Proportion of (a) eggs and (b) average number of spermatozoa/egg bound and penetrating the outer $(<1 / 2)$ and inner $(>1 / 2$ ) zona pellucida. ( $Z$ ) Ham's F10 medium; $(\square)$ PBS; $(\square)$ pentoxifylline. Pentoxifylline enhanced $(P<0.05)$ sperm binding, but zona penetration did not differ $(P>0.05)$ among groups. Values with different superscripts within categories are significantly different $(P<0.05)$.

Panthera lineage produces relatively high quality semen (Howard, 1993), and the snow leopard is no exception. In this study, the rapid decline in snow leopard sperm motility ratings in vitro appeared to be associated primarily with culture conditions. Whereas sperm motility in the tiger (also in the Panthera genus) is supported in vitro by Ham's F10 (for as long as $6 \mathrm{~h}$; Donoghue et al., 1990, 1992c), this medium abruptly killed snow leopard spermatozoa.

A variety of culture media, both simple and complex, supports longevity of spermatozoa and IVF in domestic cats (Bowen, 1977; Goodrowe et al., 1988; Johnston et al., 1991a), and a high fertilization rate ( $80 \%)$ can be achieved in Ham's FIO (Johnston et al., 1991a). Ham's F10 medium has been chosen for IVF for several felids including the cheetah (Donoghue et al., 1992a), tiger (Donoghue et al., 1990), puma and lion (Johnston et al., 1991b). Therefore, it was interesting that P. uncia spermatozoa were compromised by Ham's F10, an adverse response indicating a species-specific sensitivity not previously observed among felids.
Longevity responses of spermatozoa were compared using media containing different constituents and levels of complexity to identify a supportive medium for snow leopard spermatozoa and to begin defining the detrimental component(s) in Ham's F10. RPMI-1640, another complex medium, has only slight differences in composition compared with Ham's F10. Both included similar inorganic salts and amino acids with only one or two different components of each type. The osmolalities of RPMI and Ham's F10 were also similar (279 and $285 \pm 5 \%$, respectively), but the $\mathrm{pH}$ differed (8.1 and 7.3, respectively). HTF was 'simple', containing no amino acids or vitamins, but having energy sources that were absent from PBS, the simplest medium tested. It was not surprising that RPMI failed to prolong sperm motility given its similarity to Ham's F10. However, we anticipated a potential benefit because the $\mathrm{pH}$ of RPMI was similar to snow leopard semen (8.6 \pm 0.1 ). Nevertheless, there was no overall difference in sperm motility profiles between Ham's F10 and RPMI, supporting the hypothesis that complex media are deleterious to snow leopard spermatozoa. The marked difference in supportive capabilities between PBS and HTF was also surprising considering HTF contained only four components (magnesium sulfate, sodium bicarbonate, glucose and sodium lactate) that were not present in PBS. Even the $\mathrm{pH}$ and osmolality of PBS and HTF were similar $\quad(7.5 \pm 0.2$ and $7.3 \pm 0.2 ; 289 \pm 14$ and $280 \pm 8$ mosmol, respectively). This information will provide the basis for designing more detailed studies to identify the media component(s) detrimental to snow leopard sperm viability.

Although PBS sustained snow leopard sperm motility for up to $6 \mathrm{~h}$ in vitro, the sperm motility index decreased by $66 \%$ during the incubation. This degree of decline was much more severe than that reported for domestic cat (Howard et al., 1990, 1993) or for tiger (Donoghue et al., 1992c) spermatozoa in Ham's F10. However, in those studies, spermatozoa were washed free of seminal fluid, then resuspended in Ham's F10 and evaluated in vitro. Washing felid spermatozoa prolongs their motility in vitro (Howard et al., 1990; Howard, 1993), which was the justification for analysing the impact of washing on longevity of snow leopard spermatozoa. Findings were similar to results in other felids, indicating that seminal fluid of snow leopard is detrimental to sperm motility. There is also growing evidence that spermatozoa from a variety of nondomestic species maintain motility longer in vitro if they are held at ambient rather than body temperature (Howard et al. 1986b; Howard and Wildt, 1990; Schiewe et al., 1991). This difference was not evident in snow leopards, but the absence of $\mathrm{CO}_{2}$ in the atmosphere of samples held at $23^{\circ} \mathrm{C}$ may have been a confounding factor. The effect of holding temperature on longevity of snow leopard spermatozoa may become pronounced only when an optimal culture medium is identified.

Attempts to enhance sperm motility in humans have included supplementing culture media with metabolic promoting substances such as cAMP (Tash and Means, 1982), caffeine (Moussa, 1983) and pentoxifylline (Rees et al., 1990; Yovich et al., 1990). The usual effect is improved overall sperm motility and rate of progressive motility. In the study reported here, we examined pentoxifylline at a concentration effective in humans ( $1 \mathrm{mg} \mathrm{ml}{ }^{-1}$; Yovich ef al., 1990). Pentoxifylline increased the overall sperm motility index of snow leopards, indicating that 


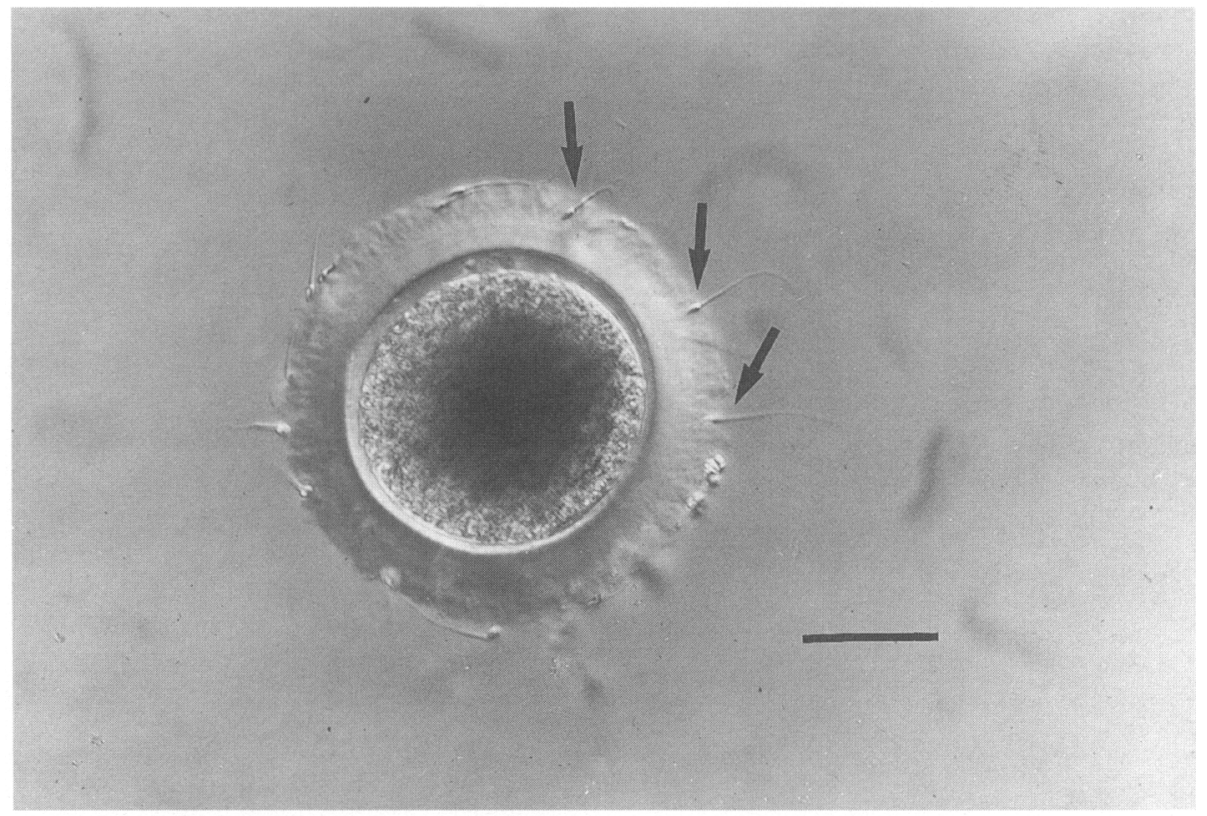

Fig. 4. Domestic cat egg with snow leopard spermatozoa bound to and penetrating the outer zona pellucida. Several spermatozoa are observed immediately adjacent to the inner-outer zona interface (arrows). Scale bar represents $50 \mu \mathrm{m}$.

the metabolism of snow leopard spermatozoa, like that of human spermatozoa, is sensitive to this metabolic stimulator. None the less, the enhancer could not prevent the steady, rather rapid decline of sperm motility during the $6 \mathrm{~h}$ incubation and, as in humans (Sikka and Hellstrom, 1991; Tournaye et al., 1993), failed to affect the ability of spermatozoa to penetrate the zona pellucida.

The cat zona pellucida is composed of a phenotypically distinct bilayer (Andrews et al., 1992). Snow leopard spermatozoa readily bound and penetrated the outer layer of this zona pellucida, as do leopard cat (Howard and Wildt, 1990) and tiger (Donoghue et al., 1992c) spermatozoa co-cultured with domestic cat eggs. However, snow leopard spermatozoa could not penetrate the inner zona layer of these heterologous eggs. Penetration failure in the Ham's F10 group was not surprising because sperm motility was poor by $6 \mathrm{~h}$ in vitro. Previous studies in cheetahs have demonstrated that a severe loss of sperm motility within $6 \mathrm{~h}$ is associated with failure of egg penetration (Donoghue et al., 1992a). Snow leopard spermatozoa in PBS or PBSP maintained motility for $6 \mathrm{~h}$, but failed to penetrate the inner zona of cat eggs. It is therefore possible that failed zona penetration in PBS and PBSP treatments was related to cross-species incompatibility between $P$. uncia spermatozoa and $F$. catus eggs. The strongest evidence supporting this hypothesis was the successful penetration of homologous eggs in the conspecific IVF attempts which also clearly demonstrated that the PBS culture conditions were sufficient to support snow leopard sperm-egg interaction.

The incompatibility between snow leopard spermatozoa and domestic cat eggs appeared to lie at the inner zona interface because binding and outer zona penetration were readily achieved, yet, snow leopard sperm heads were frequently observed immediately adjacent to the inner-outer zona interface without penetrating further. The inner zona is responsible for selecting against pleiomorphic spermatozoa in felids (Howard et al., 1993), and the present results suggest that this region may also restrict penetration by populations of morphologically normal spermatozoa, perhaps by spermatozoa that fail to capacitate. Although capacitation of domestic cat, leopard cat and cheetah spermatozoa is achieved readily by supplementing medium with a protein source (Howard et al., 1991; Andrews et al, 1992), snow leopard spermatozoa may have more specific requirements, including a longer time to achieve capacitation. Homologous snow leopard eggs were co-incubated with spermatozoa for $8 \mathrm{~h}, 2 \mathrm{~h}$ more than for heterologous eggs, thus increasing the time for spermatozoa to fully achieve capacitation and zona penetration. Interaction with cumulus cells (absent from the domestic cat eggs) or cellular factors may also be important for snow leopard sperm capacitation and activation; an investigation is currently in progress.

In summary, these results confirm that there is significant variation among felids in gamete function, culture requirements and the ability of spermatozoa and eggs to interact across species. One practical consequence is that assisted reproduction (artificial insemination, IVF) for this taxon will require adaptive methods reflecting species-specific gamete characteristics. Although the snow leopard now appears unique among felids in that sperm longevity and function in vitro are supported by a simple rather than a complex medium, it is likely that other unique characteristics will be revealed as the database is established for the 37 extant species in the Felidae.

This research was supported by grants from the Phillip Reed Foundation to NOAHS (New Opportunities in Animal Health Sciences) Center, Friends of the National Zoo (FONZ), the Ralston Purina Company/Conservation Endowment Fund of the American Zoo and Aquariums Association and NIH RO1 HD 23853. The 
authors thank the Snow Leopard Species Survival Plan Coordinator, Dan Wharton of the Wildlife Conservation Park/Bronx Zoo for coordinating and supporting this research. The authors also thank the following institutions for generous assistance and willingness to collaborate: Greater Baton Rouge Zoo, Baton Rouge, LA; Jackson Zoological Park, Jackson, MS; Wildlife Conservation Park/Bronx Zoo, Bronx, NY; Seneca Park Zoo, Rochester, NY; and Woodland Park Zoological Gardens, Seattle, WA.

\section{References}

Andrews JC, Howard JG, Bavister BD and Wildt DE (1992) Sperm capacitation in the domestic cat (Felis catus) and leopard cat (Felis bengalensis) as studied with a salt-stored zona pellucida penetration assay Molecular Reproduction and Development 31 200-207

Bowen RA (1977) Fertilization in vitro of feline ova by spermatozoa from the ductus deferens Biology of Reproduction 17 144-147

Convention on International Trade in Endangered Species of Wild Flora and Fauna, part of the Endangered Species Act (1984) Code Fed Reg, part 23

Donoghue AM, Johnston LA, Seal US, Armstrong DL, Tilson RL, Wolf P, Petrini K, Simmons LG, Gross T and Wildt DE (1990) In vitro fertilization and embryo development in vitro and in vivo in the tiger (Panthera tigris) Biology of Reproduction 43 733-744

Donoghue AM, Howard JG, Byers AP, Goodrowe KL, Bush M, Blumer E, Lukas J, Stover J, Snodgrass K and Wildt DE (1992a) Correlation of sperm viability with gamete interaction and fertilization in vitro in the cheetah (Acinonyx jubatus) Biology of Reproduction 46 1047-1056

Donoghue AM, Johnston LA, Munson L, Brown JL and Wildt DE (1992b) Influence of gonadotropin treatment interval on follicular maturation, in vitro fertilization, circulating steroid concentrations and subsequent luteal function in the domestic cat Biology of Reproduction 46 972-980

Donoghue AM, Johnston LA, Seal US, Armstrong DL, Simmons LG, Gross T, Tilson RL, Wolf P and Wildt DE (1992c) Ability of thawed tiger (Panthera tigris) spermatozoa to fertilize conspecific eggs and bind and penetrate domestic cats egss in vitro Journal of Reproduction and Fertility 96 555-564

Goodrowe KL, Wall RJ, O'Brien SJ, Schmidt PM and Wildt DE (1988) Developmental competence of domestic cat follicular oocytes after fertilization in vitro Biology of Reproduction 39 355-372

Howard JG (1993) Semen collection and analysis in carnivores Zoo and Wild Animal Medicine pp 390-399 Ed. ME Fowler. WB Saunders Co., Philadeiphia

Howard JG and Wildt DE (1990) Ejaculate and hormonal characteristics in the leopard cat (Felis bengalensis), and sperm function as measured by in vitro penetration of zona-free hamster ova and zona-intact domestic cat oocytes Molecular Reproduction and Development 26 163-174

Howard JG, Bush M, Wildt DE (1986a) Semen collection, analysis and cryopreservation in nondomestic mammals Current Therapy in Theriogenology, pp 1047-1053 Ed. DA Morrow. WB Saunders Co., Philadelphia

Howard JG, Bush M, de Vos V, Schiewe MC, Pursel VG and Wildt DE (1986b) Influence of cryoprotective diluent on post-thaw viability and acrosomal integrity of spermatozoa of the African elephant (Loxodonta africana) Joumal of Reproduction and Fertility 78 295-306

Howard JG, Brown JL, Bush M and Wildt DE (1990) Teratospermic and normospermic domestic cats: ejaculate traits, pituitary-gonadal hormones and improvement of spermatozoal motility and morphology after swim-up processing Journal of Andrology $11204-215$
Howard JG, Barone MA, Bush M and Wildt DE (1991) A heterologous saltstored zonae pellucidae assay for assessing sperm capacitation and the impact of teratospermia in the cheetah (Acinonyx jubatus) Journal of Andrology 12 (Supplement) P-50

Howard JG, Donoghue AM, Johnston LA and Wildt DE (1993) Zona pellucida filtration of structurally-abnormal spermatozoa and reduced fertilization in teratospermic cats Biology of Reproduction 49, 131-139

Johnston LA, O'Brien SJ and Wildt DE (1989) In vitro maturation and fertilization of domestic cat follicular oocytes Gamete Research 24 343-356

Johnston LA, Donoghue AM, O'Brien SJ and Wildt DE (1991a) Culture medium and protein supplementation influence in vitro fertilization and embryo development in the domestic cat Joumal of Experimental Zoology 257 350-359

Johnston LA, Donoghue AM, O'Brien SJ and Wildt DE (1991b) Rescue and maturation in vitro of follicular oocytes collected from nondomestic felid species Biology of Reproduction 45 898-906

Moussa MM (1983) Caffeine and sperm motility Fertility and Sterility 39 845-848

Rees JM, Ford WCL and Hull MGR (1990) Effect of caffeine and of pentoxifylline on the motility and metabolism of human spermatozoa Journal of Reproduction and Fertility 90 147-156

SAS (1985) SAS User's Guide Statistical Analysis Systems Institute, Inc., Cary, NC

Schiewe MC, Bush M, de Vos V, Brown JL and Wildt DE (1991) Semen characteristics, sperm freezing and endocrine profiles in free-living wildebeest (Connochaetes taurinus) and greater kudu (Tragelaphus strepsiceros) Journal of Zoo and Wildlife Medicine 22 58-72

Sikka SC and Hellstrom WJG (1991) Functional evaluation and motility parameters of pentoxifylline-stimulated cryopreserved human sperm Assisted Reproductive Technology/Andrology 1 309-319

Tash JS and Means AR (1982) Regulation of protein phosphorylation and motility of sperm by cAMP and calcium Biology of Reproduction 26 745-763

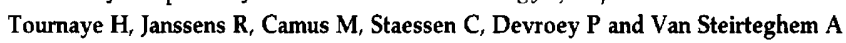
(1993) Pentoxifylline is not useful in enhancing sperm function in cases with previous in vitro fertilization failure Ferfility and Sterility 59 210-215

Wildt DE, Bush M, Howard JG, O'Brien SJ, Meltzer D, van Dyk A, Ebedes $\mathrm{H}$ and Brand DJ (1983) Unique seminal quality in the South African cheetah and a comparative evaluation in the domestic cat Biology of Reproduction 29 1019-1025

Wildt DE, Howard JG, Hall LL and Bush M (1986) Reproductive physiology of the clouded leopard: I. Electroejaculates contain high proportions of pleiomorphic spermatozoa throughout the year Biology of Reproduction 34 937-947

Wildt DE, Donoghue AM, Johnston LA, Schmidt PM and Howard JG (1992a) Species and genetic effects on the utility of biotechnology for conservation Biotechnology and the Conservation of Genetic Diversity pp 45-61 Eds HDM Moore, WV Holt and GM Mace. Clarendon Press, Oxford

Wildt DE, Monfort SL, Donoghue AM, Johnston LA and Howard JG (1992b) Embryogenesis in conservation biology - or, how to make an endangered species embryo Theriogenology 37 161-184

Yovich JM, Edirisinghe WR, Cummins JM and Yovich JL (1990) Influence of pentoxifylline in severe male factor infertility Fertility and Sterility $\mathbf{5 3}$ 715-721 